\title{
Dynamic stability of a curved pipe bent in the arc of a circle on hinge supports at the ends
}

\author{
D S LOLOV and S V LILKOVA-MARKOVA \\ Department of Technical Mechanics, University of Architecture, 1046, Bulgaria, \\ Sofia \\ e-mail: dlolov@yahoo.com; lilkova_fhe@uacg.bg \\ MS received 12 September 2005; revised 29 April 2006
}

\begin{abstract}
Curved pipes conveying fluids are investigated in the paper. Methods of numerical solution of the dynamic stability of a pipe in its plane are developed. An example of a curved pipe is solved by these methods. A non-dimensional parameter of flow velocity and a non-dimensional circular frequency are obtained.
\end{abstract}

Keywords. Curved pipe; flutter loss of stability.

\section{Introduction}

Most scientific researches in the area of vibrations of pipes conveying liquids are concerned with investigation of their rectilinear sections. The question about dynamic stability of curved sections of pipelines arises naturally. This is the theme of our broad investigation in the last few years.

Vibrations of pipes outlined on a plane curve are in the plane as well as out of it. The outof-the-plane dynamics of curved pipes conveying fluids in two supporting cases is considered by Lolov \& Lilkova-Markova (2005).

Investigation of vibrations in the plane of the pipes is considerably more complicated and expensive. Methods of reaching the numerical solution of dynamic stability along these lines are developed in this paper.

\section{Formulation of the problem}

The pipe under investigation, supported on joints, bent in the form of an arc of a circle with radius $R$ is shown in figure 1. The length of the arc is $s_{f}$. The material of the pipe is linear elastic with Young's modulus, $E$. The cross-sectional parameters are area $A$ and axis moment of inertia, $I$. The fluid flowing in the pipe with velocity $U$ is heavy and noncompressible. 


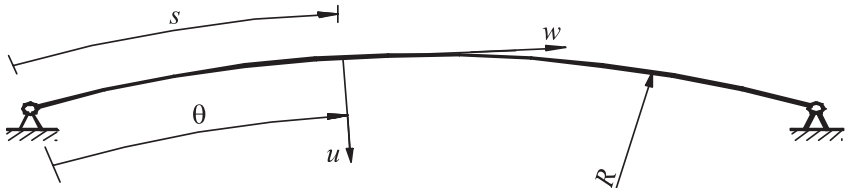

Figure 1. Schematic and parameters of the curved pipe.

The differential equations describing the vibration of the pipe in its plane are written about the axes of the natural coordinate system:

$$
\begin{aligned}
& -E I\left(\frac{\partial^{4} u}{\partial s^{4}}+\frac{1}{R} \frac{\partial^{3} w}{\partial s^{3}}\right)+\frac{E A}{R}\left(\frac{\partial w}{\partial s}-\frac{u}{R}\right)-M U^{2}\left(\frac{\partial^{2} u}{\partial s^{2}}+\frac{1}{R} \frac{\partial w}{\partial s}\right) \\
& -2 M U\left(\frac{\partial^{2} u}{\partial s \partial t}+\frac{1}{R} \frac{\partial w}{\partial t}\right)-(M+m) \frac{\partial^{2} u}{\partial t^{2}}=0, \\
& \frac{E I}{R}\left(\frac{\partial^{3} u}{\partial s^{3}}+\frac{1}{R} \frac{\partial^{2} w}{\partial s^{2}}\right)+E A\left(\frac{\partial^{2} w}{\partial s^{2}}-\frac{1}{R} \frac{\partial u}{\partial s}\right) \\
& \quad+M U\left(\frac{1}{R} \frac{\partial u}{\partial t}-\frac{\partial^{2} w}{\partial s \partial t}\right)-(M+m) \frac{\partial^{2} w}{\partial t^{2}}=0,
\end{aligned}
$$

where $t$ is the time, $u$ is the displacement on the normal of the curve and $w$ that on the tangent, and $s$ is the curvilinear abscissa. The mass of the pipe per unit length is denoted by $m$ and the mass of fluid per unit length of the pipe by $M$.

\section{Methods of solution}

For convenient reasons, the curvilinear abscissa $s$ in (1) and (2) is expressed by the central angle $\theta$. The following equations are thus obtained,

$$
\begin{aligned}
& \frac{E I}{R^{3}}\left(\frac{\partial^{4} u}{\partial \theta^{4}}+\frac{\partial^{3} w}{\partial \theta^{3}}\right)-\frac{E A}{R}\left(\frac{\partial w}{\partial \theta}-u\right)+\frac{M U^{2}}{R^{3}}\left(\frac{\partial^{2} u}{\partial \theta^{2}}+\frac{\partial w}{\partial \theta}\right) \\
& \quad+2 M U\left(\frac{\partial^{2} u}{\partial \theta \partial t}+\frac{\partial w}{\partial t}\right)=(M+m) R \frac{\partial^{2} u}{\partial t^{2}} \\
& \frac{E I}{R^{3}}\left(\frac{\partial^{3} u}{\partial \theta^{3}}+\frac{\partial^{2} w}{\partial \theta^{2}}\right)+\frac{E A}{R}\left(\frac{\partial^{2} w}{\partial \theta^{2}}-\frac{\partial u}{\partial \theta}\right) \\
& \quad+M U\left(\frac{\partial u}{\partial t}-\frac{\partial^{2} w}{\partial \theta \partial t}\right)=(M+m) R \frac{\partial^{2} w}{\partial t^{2}} .
\end{aligned}
$$

The following non-dimensional quantities are included:

$$
\bar{u}=\frac{u}{R} ; \quad \bar{w}=\frac{w}{R} ; \quad \bar{t}=\frac{t}{T_{o}} ; \quad \bar{U}=U s_{f}\left(\frac{M}{E I}\right)^{1 / 2},
$$

where $s_{f}$ is the length of the pipe and $T_{o}=R^{2}[(M+m) / E I]^{1 / 2}$. 
After replacing $k^{2}=I / A R^{2}$, we get the equations of motion of the pipe in its plane are,

$$
\begin{aligned}
& k^{2}\left(\frac{\partial^{4} \bar{u}}{\partial \theta^{4}}+\frac{\partial^{3} \bar{w}}{\partial \theta^{3}}\right)-\left(\frac{\partial \bar{w}}{\partial \theta}-\bar{u}\right)+\frac{k^{2} \bar{U}^{2}}{s_{f}^{2}}\left(\frac{\partial^{2} \bar{u}}{\partial \theta^{2}}+\frac{\partial \bar{w}}{\partial \theta}\right) \\
& +2 k^{2} R\left[\frac{M}{(M+m)}\right]^{1 / 2} \bar{U}\left(\frac{\partial^{2} \bar{u}}{\partial \theta \partial \bar{t}}+\frac{\partial \bar{w}}{\partial \bar{t}}\right)=k^{2} \frac{\partial^{2} \bar{u}}{\partial \bar{t}^{2}}, \\
& k^{2}\left(\frac{\partial^{3} \bar{u}}{\partial \theta^{3}}+\frac{\partial^{2} \bar{w}}{\partial \theta^{2}}\right)+\left(\frac{\partial^{2} \bar{w}}{\partial \theta^{2}}-\frac{\partial \bar{u}}{\partial \theta}\right) \\
& \quad+2 k^{2} R\left[\frac{M}{(M+m)}\right]^{1 / 2} \bar{U}\left(\frac{\partial \bar{u}}{\partial \bar{t}}-\frac{\partial^{2} \bar{w}}{\partial \theta \partial \bar{t}}\right)=k^{2} \frac{\partial^{2} \bar{w}}{\partial \bar{t}^{2}} .
\end{aligned}
$$

The solutions of these differential equations are obtained in the form:

$$
\bar{u}(\theta, \bar{t})=C_{u} e^{i(\gamma \theta-\Omega \bar{t})} ; \quad \bar{w}(\theta, \bar{t})=C_{w} e^{i(\gamma \theta-\Omega \bar{t})},
$$

where $\gamma$ and $\Omega$ are non-dimensional wave numbers and circular frequencies. They are expressed by the real ones.

$$
\gamma=R \Gamma ; \quad \Omega=\omega T_{o}
$$

The amplitudes $C_{u}$ and $C_{w}$ in (8) are unknown integrating constants that are not dependent. The connection between them is expressed in this way:

$$
\alpha=\frac{C_{u}}{C_{w}}=\frac{k^{2} \gamma^{4}+1-\left(k^{2} \bar{U}^{2} \gamma^{2} / s_{f}^{2}\right)+J \Omega \gamma+k^{2} \Omega^{2}}{k^{2} \gamma^{3} i+\gamma i+J \Omega i},
$$

where $J=2 k^{2} R[M /(M+m)]^{1 / 2} \bar{U}$.

The following homogeneous system linear algebraic equations of constants $C_{u}$ and $C_{w}$ is obtained on substituting (8) in differential equations (6) \& (7):

$$
\left|\begin{array}{ll}
-k^{2} \gamma^{3} i-\gamma-\frac{k^{2} \bar{U}^{2} \gamma i}{s_{f}^{2}}-J \omega i & k^{2} \gamma^{4}+1-\frac{k^{2} \bar{U}^{2} \gamma^{2}}{s_{f}^{2}}+J \omega \gamma+k^{2} \omega^{2} \\
-k^{2} \gamma^{2}-\gamma^{2}-J \gamma \omega+k^{2} \omega^{2} & -k^{2} \gamma^{3} i-\gamma i-J \omega i
\end{array}\right|\left\{\begin{array}{l}
C_{w} \\
C_{u}
\end{array}\right\}=0 .
$$

The determinant of the coefficients in front of them is set to zero for a non-zero solution of the system. So the characteristic equation for non-dimensional wave numbers $\gamma$ is obtained:

$$
\begin{aligned}
& k^{2} \gamma^{6}+\left(-k^{2} J \Omega\right) \gamma^{5}+k^{2}\left(i-1-\frac{\bar{U}^{2}\left(2 k^{2}+1\right)}{s_{f}^{2}}-k^{2} \Omega^{2}\right) \gamma^{4}+J \Omega\left(1+k^{2}\left(\frac{\bar{U}^{2}}{s_{f}^{2}}-1\right)\right) \gamma^{3} \\
& +\left(1+i-k^{2}\left(\frac{\bar{U}^{2}}{s_{f}^{2}}-1\right)+\left(k^{4}\left(1+\frac{\bar{U}^{2}}{s_{f}^{2}}\right)+k^{2}+J^{2}\right) \Omega^{2}\right) \gamma^{2}+J \Omega i\left(1+\frac{k^{2} \bar{U}^{2}}{s_{f}^{2}}\right) \gamma \\
& -\left(J+k^{2}+k^{4} \Omega^{2}\right) \Omega^{2}=0 .
\end{aligned}
$$


The following boundary equations are described for the pipe in figure 1:

$$
\begin{aligned}
& \bar{u}(0, \bar{t})=0 ; \quad \bar{u}\left(\frac{s_{f}}{R}, \bar{t}\right)=0 ; \quad \bar{w}(0, \bar{t})=0 ; \quad \bar{w}\left(\frac{s_{f}}{R}, \bar{t}\right)=0 ; \\
& M(0, \bar{t})=0 ; \quad M\left(\frac{s_{f}}{R}, \bar{t}\right)=0 .
\end{aligned}
$$

The differential equation of the elastic line of a beam outlined on the circle is:

$$
M=-E I\left(\frac{\mathrm{d}^{2} u}{\mathrm{~d} s^{2}}+\frac{u}{R^{2}}\right) .
$$

The boundary conditions are:

$$
-E I \frac{\mathrm{d}^{2} u}{\mathrm{~d} s^{2}}=0, \text { for } s=0 \text { and for } s=s_{f},
$$

and therefore

$$
\frac{\mathrm{d}^{2} \bar{u}}{\mathrm{~d} s^{2}}=0, \text { for } s=0 \text { and for } s=s_{f} .
$$

Then the conditions (14) for $\theta=0$ and for $\theta=s_{f} / R$ take the form:

$$
\begin{aligned}
& \bar{u}(\theta, \bar{t})=C_{u} e^{i(\gamma \theta-\Omega \bar{t})}=0 ; \\
& \bar{w}(\theta, \bar{t})=C_{w} e^{i(\gamma \theta-\Omega \bar{t})}=0 ; \\
& \frac{\mathrm{d}^{2} \bar{u}}{\mathrm{~d} s^{2}}=-\frac{C_{u} \gamma^{2}}{R} e^{i(\gamma \theta-\Omega \bar{t})}=0 .
\end{aligned}
$$

In the case of real roots $\gamma$ of the characteristic equation (13), the functions $\bar{w}(\theta, \bar{t})$ and $\bar{u}(\theta, \bar{t})$ are expressed in the following manner:

$$
\begin{aligned}
& \bar{w}(\theta, \bar{t})=\left(\begin{array}{c}
C_{w 1}^{+} e^{-i \gamma_{1} \theta}+C_{w 2}^{+} e^{-i \gamma_{2} \theta}+C_{w 3}^{+} e^{-i \gamma_{3} \theta} \\
+C_{w 1}^{-} e^{i \gamma_{1} \theta}+C_{w 2}^{-} e^{i \gamma_{2} \theta}+C_{w 3}^{-} e^{i \gamma_{3} \theta}
\end{array}\right) e^{-i \Omega \bar{t}} \\
& \bar{u}(\theta, \bar{t})=\left(\begin{array}{c}
C_{u 1}^{+} e^{-i \gamma_{1} \theta}+C_{u 2}^{+} e^{-i \gamma_{2} \theta}+C_{u 3}^{+} e^{-i \gamma_{3} \theta} \\
+C_{u 1}^{-} e^{i \gamma_{1} \theta}+C_{u 2}^{-} e^{i \gamma_{2} \theta}+C_{u 3}^{-} e^{i \gamma_{3} \theta}
\end{array}\right) e^{-i \Omega \bar{t}} .
\end{aligned}
$$

These functions are replaced in conditions (18), (19) and (20). So a homogeneous system of linear algebraic equations is obtained for the parameters $C_{u}$ and $C_{w}$. For a non-zero solution the determinant of the coefficients before them must to be equal to zero.

From (18) the multipliers in front of $C_{u}$ are:

$$
\begin{array}{llllll}
\alpha & \alpha & \alpha & \alpha & \alpha & \alpha,
\end{array}
$$

and

$$
\alpha e^{-i \gamma_{1}^{\frac{s_{f}}{R}}} \alpha e^{-i \gamma_{2}{ }^{\frac{s_{f}}{R}}} \alpha e^{-i \gamma_{3}^{\frac{s_{f}}{R}}} \alpha e^{i \gamma_{1}^{\frac{s_{f}}{R}}} \alpha e^{i \gamma_{2}^{\frac{s_{f}}{R}}} \alpha e^{i \gamma_{3}{ }^{\frac{s_{f}}{R}}} .
$$


From (19), the multipliers in front of $C_{w}$ are:

$$
\begin{array}{llllll}
1 & 1 & 1 & 1 & 1 & 1
\end{array}
$$

and

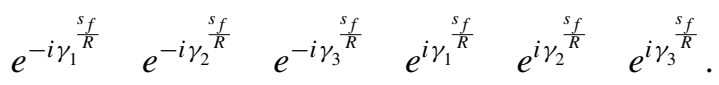

From (20), the multipliers in front of $C_{u}$ are:

$$
\begin{array}{llllll}
\alpha \gamma_{1}^{2} & \alpha \gamma_{2}^{2} & \alpha \gamma_{3}^{2} & \alpha \gamma_{1}^{2} & \alpha \gamma_{2}^{2} & \alpha \gamma_{3}^{2}
\end{array}
$$

and

$$
\alpha \gamma_{1}^{2} e^{-i \gamma_{1}^{\frac{s_{f}}{R}}} \quad \alpha \gamma_{2}^{2} e^{-i \gamma_{2}^{\frac{s_{f}}{R}}} \quad \alpha \gamma_{3}^{2} e^{-i \gamma_{3}^{\frac{s_{f}}{R}}} \quad \alpha \gamma_{1}^{2} e^{i \gamma_{1}^{\frac{s_{f}}{R}}} \quad \alpha \gamma_{2}^{2} e^{i \gamma_{2}^{\frac{s_{f}}{R}}} \quad \alpha \gamma_{3}^{2} e^{i \gamma_{3}^{\frac{s_{f}}{R}}} .
$$

The flow velocity $U$ and the circular frequency $\Omega$ take part in the elements of the determinant. The solution is reached in the following way: The flow velocity and the circular frequency vary for a given pipe. The wave numbers $\gamma$ are obtained for two pairs of $U$ and $\Omega$. The values of $U$ and $\Omega$ at which the determinant is equal to zero are the ones for the flutter loss of stability. In the investigation we considered the smallest of the values of the flow velocity and the respective $\omega$.

\section{Numerical results}

A numerical example is solved to show the developed method. The pipe considered was on hinge supports at the ends. The cross-section properties are: inner radius $9.5 \mathrm{~cm}$ and outer radius $10 \mathrm{~cm}$. The pipe is bent in the shape of an arc of a circle with radius $R=20 \mathrm{~m}$ and a central angle $\theta_{\max }=60^{\circ}$. The material of the pipe is steel with the Young's modulus $E=20600 \mathrm{kN} / \mathrm{cm}^{2}$.

The flutter loss of stability is characterized with a non-dimensional parameter of flow velocity $\bar{U}=4.92$ and with non-dimensional circular frequency $\Omega=19,35$. This result is compared with that obtained by Lolov \& Lilkova-Markova (2006) by matrix method. There is good coincidence.

\section{Conclusion}

The proposed method is expensive to implement and is suitable only for pipes with constant rigidity $E I$.

\section{References}

Kanga B, Riedelb C H, Tanc C A 2003 Free vibration analysis of planar curved beams by wave propagation. J. Sound Vibr. 260: 19-44

Lolov D S, Lilkova-Markova S V 2005 Free vibrations out of the plane of curved plane pipe conveying fluid in two case of supporting. Annual of the University of Architecture, Civil Engineering and Geodesy 42: 96-101

Lolov D S, Lilkova-Markova S V 2006 Determination of the critical flow velocity of fluid conveying in curved pipe outlined on a circumference. Annual of the University of Architecture, Civil Engineering and Geodesy 42: 124-129 